\title{
Validación de la Escala de Reacciones ante Experiencias de Trato Desigual en Salud
}

\section{Validating the Scale of Reactions to Experiences of Unequal Treatment in Health}

\author{
Validação da Escala de Reações ante \\ Experiências de Trato Desigual em Saúde
}

\author{
María José Baeza-Rivera, PhD* \\ Natalia Salinas-Oñate, $\mathrm{PhD}^{2,3}$ \\ Neli Escandón-Nagel, PhD 1,4 \\ Esteban Caamaño Mardones, Mg. ${ }^{5}$
}

Recibido: 31 de marzo de $2020 \cdot$ Aceptado: 28 de julio de 2020

Doi: https://doi.org/10.12804/revistas.urosario.edu.co/revsalud/a.9798

Para citar este artículo: Baeza-Rivera MJ, Salinas-Oñate N, Escandón Nagel N, Caamaño Mardones E. Validación de la Escala de

Reacciones ante Experiencias de Trato Desigual en Salud. Rev Cienc Salud. 2020;18(3):1-15. https://doi.org/10.12804/revistas.urosario. edu.co/revsalud/a.9798

\section{Resumen}

Introducción: este artículo tiene por objetivo estimar las propiedades psicométricas de la Escala de Reacciones ante Experiencias de Trato Desigual en Salud de Baeza-Rivera (2015) y establecer si existen diferencias estadísticamente significativas entre quienes se atienden en el sistema privado y público y la pertenencia o no a la etnia mapuche. Materiales y métodos: participaron 337 personas cuyo promedio de edad fue de 31 años, en su mayoría mujeres. El 57\% reportó ser usuario del sistema público de salud y el $32 \%$ declaró su pertenencia a la etnia mapuche. Se realizó un análisis factorial exploratorio, análisis de confiabilidad y Anova factorial. Resultados: los hallazgos dan cuenta de un instrumento con adecuadas propiedades psicométricas, que puede usarse en población con características similares a la muestra empleada. Además, se observa que existen diferencias estadísticamente significativas en función del sistema de salud en que se atiende la persona y la pertenencia a la etnia mapuche. Conclusiones: el instrumento tiene adecuadas propiedades psicométricas que presenta dos factores relacionados, que sirve para ser utilizado en contextos de salud, lo que permite comprender los procesos de salud-enfermedad.

Palabras clave: propiedades psicométricas; sistema de salud; etnia.

1 Universidad Católica de Temuco (Chile).

Autora de correspondencia: maria.baeza@uct.cl oRciD: https://orcid.org/0000-0001-7763-0148

2 Universidad de La Frontera (Chile).

3 ORCID: https://orcid.org/0000-0001-6451-4965

4 ORCID: https://orcid.org/0000-0002-6697-2692

5 ORCID: https://orcid.org/0000-0003-4390-9751 
Introduction: This study aimed to estimate the psychometric properties of the Scale of Reactions to Experiences of Unequal Treatment in Health, from Baeza-Rivera (2015), and to determine any statistically significant differences between those using the private and public healthcare systems and those belonging to the Mapuche ethnic group. Materials and Methods: The Scale of Reactions to Experiences of Unequal Treatment in Health was used in 337 participants (mostly women) with a mean age of 31 years. Of these, 57\% reportedly used the public healthcare system and 32\% belonged to the Mapuche ethnic group. Subsequently, exploratory factor analysis and reliability analysis using Cronbach's alpha and factorial analysis of variance were conducted. Results: The findings demonstrated that this scale has ideal psychometric properties, such as reliability and factorial validity, and it can be used in a population with characteristics similar to those of the sample population used in this study. Additionally, statistically significant differences were observed between those using the healthcare systems and those belonging to the Mapuche ethnic group. Conclusions: This scale shows adequate psychometric properties, such as the two related factors reliability and factorial validity, and can be used in health-related contexts, thus allowing for an understanding of health-disease processes.

Keywords: Psychometric; healthcare system; ethnicity; discrimination.

\section{Resumo}

Introdução: este artigo tem por objetivo estimar as propriedades psicométricas da Escala de Reações ante Experiências de Trato Desigual em Saúde, de Baeza-Rivera (2015), e estabelecer se existem diferenças estatisticamente significativas entre quem se atendem no sistema privado e público e a pertença ou não à etnia mapuche. Materiais e métodos: participaram 337 pessoas cuja média de idade foi de 31 anos, em sua maioria mulheres, 57\% reportou ser usuário do sistema público de saúde e $32 \%$ declarou pertencer à etnia mapuche. Se realizou análise fatorial exploratório, análise de confiabilidade e a Anova fatorial. Resultados: se conta com um instrumento com adequadas propriedades psicométricas, que pode ser utilizado em população com características similares à amostra utilizada. Adicionalmente, se observa que existem diferenças estatisticamente significativas em função do sistema de saúde em que se atende a pessoa e a pertença ou não a etnia mapuche. Conclusões: o instrumento tem adequadas propriedades psicométricas, apresenta dois fatores relacionados, e serve para ser utilizado em contextos de saúde, permitindo compreender os processos de saúde-doença.

Palavras-chave: propriedades psicométricas; sistema de saúde; etnia.

\section{Introducción}

as reformas sanitarias acaecidas en los últimos veinte años en América Latina y el Caribe,
impulsadas por la Organización Mundial de la Salud, en pos de disminuir las desigualdades en el acceso y calidad de los servicios sanitarios, han contribuido a mejorar los indicadores de salud general. Particularmente en Chile, esto se hace patente, por ejemplo, en la tasa de mortalidad infantil en menores de cinco años de edad, la cual pasó de 157 a 7 por cada 1000 habitantes desde la década de los sesenta a la actualidad (1).

Considerando lo anterior, y a fin de lograr la disminución de las inequidades de salud, se hace necesario facilitar el acceso a los sistemas sanitarios y, además, garantizar la calidad de la atención ofrecida. Por ello, en el ámbito local se han implementado diversas iniciativas, 
como el Programa de Inmunización Infantil, las Garantías Explícitas en Salud, el Programa Chile Crece Contigo, programas especiales según rango etario y aumento general de la oferta de salud. Todo ello tiene como objetivo contribuir, además, a la satisfacción de necesidades de salud de manera deliberada y democrática (2).

En este sentido, una de las estrategias más relevantes para combatir la desigualdad originada en la primera infancia ha sido el Programa Nacional de Salud en la Infancia, el cual crea condiciones para que todos los niños y las niñas crezcan en entornos favorables para un óptimo desarrollo psicosocial en la atención primaria (3). Una de sus líneas de acción más importantes es el Control del Niño Sano, una actividad dirigida a promover de forma integral la salud de los niños y a detectar oportunamente cualquier anomalía o enfermedad que pudieran presentar, para lograr su derivación oportuna (4,5). Este control es muy relevante, ya que representa el primer acercamiento de la población hacia el sistema de salud (6).

A pesar de que estos programas han tenido un impacto positivo en cuanto a indicadores globales de salud, existe un ámbito que se encuentra menos estudiado: la calidad de la atención. Al respecto, los resultados de un estudio sobre satisfacción y calidad usuaria en Chile mostraron que el $47 \%$ de los usuarios entrega razones espontáneas de insatisfacción, las cuales se distribuyen principalmente entre los largos tiempos de espera, la mala disposición por parte de los profesionales de la salud, la escasa dotación de personal, las atenciones de corta duración y la mala atención entregada (7). Asimismo, otro estudio dio cuenta de que el $45 \%$ de las personas se encuentra insatisfecha con los servicios de salud, en específico la calidad de la atención recibida por el personal administrativo, personal técnico paramédico y personal profesional (8).

Lo mencionado demuestra que, a pesar de las mejoras implementadas en el sistema de salud en Chile, hoy en día todavía existen cifras que apuntan directamente a una evaluación negativa de los servicios y atención en salud (8). Esto es de suma relevancia, pues tanto el trato recibido como la relación médico-paciente son elementos clave en la obtención de buenos resultados en salud. En cuanto a esto, los hallazgos señalan que experiencias positivas ayudan a la adherencia a los tratamientos y a las indicaciones médicas, lo cual facilita que las personas adquieran un rol activo en pos de mejorar su salud (9). Del mismo modo, la percepción por parte del paciente de un trato injusto o insatisfactorio en el ámbito sanitario podría tener consecuencias negativas tanto para la salud mental como para la física. Por ejemplo, en el plano físico se han observado problemas de presión arterial y de los propios resultados en salud, como la mortalidad (10). Por otra parte, en relación con la salud mental, se ha visto que las personas que indican haberse sentido discriminadas presentan mayor depresión, manía/hipomanía, trastornos de estrés postraumático, trastorno de ansiedad generalizada, fobia social y estrés (11-14).

Además de las consecuencias físicas y mentales, el trato injusto también impacta en la manifestación de conductas de cuidado de la salud, que puede disminuirlas, lo que se 
traduce, por ejemplo, en pocas conductas de cuidado y acceso a las prestaciones médicas, menor adherencia terapéutica, escasa asistencia a las citas médicas, menor disposición a utilizar los servicios preventivos de salud y sustitución de la atención convencional por la medicina alternativa (15-22).

En este sentido, la inversión de recursos en el ámbito sanitario debería considerar en sus políticas el abordaje de los aspectos mencionados, dado que han probado ser clave en la adherencia a los tratamientos de salud. No obstante, para ello es imprescindible contar antes con evidencia local sobre las consecuencias de los tratos injustos o discriminatorios, a partir de las cuales se detecten elementos específicos que sirvan de retroalimentación para regular el diseño y puesta en marcha de los programas sanitarios. La evidencia señala que el percibir tratos injustos o mala atención se asocia con diversas consecuencias que afectan directamente la salud de las personas, pero también afectan la forma de afrontar o responder a los tratamientos (23).

A partir de los antecedentes señalados, es relevante estudiar las reacciones experimentadas ante el trato injusto en salud, sobre todo en una de las regiones más pobres de Chile, la región de La Araucanía, que se caracteriza, precisamente, por liderar la tasa de pobreza y vulnerabilidad nacional. En específico, un $17.2 \%$ se encuentra en la pobreza, y un 4.6\%, en situación de pobreza extrema y total. Además, la región presenta mayor incidencia de pobreza multidimensional, en específico un 28.5 \% de la población de la región sufre pobreza en diferentes aspectos, como educación, salud, trabajo, seguridad social y vivienda (24). Si a esto se le suma que en la región de La Araucanía vive el 33.6 \% del total de la población indígena mapuche del país (25), grupo que ha sido históricamente vulnerado y que presenta peores indicadores de salud, educación y pobreza que la población chilena no mapuche, entonces resulta pertinente estudiar si la pertenencia o no al pueblo mapuche y el tipo de sistema en que se atiende la persona se asocian con las reacciones que puedan tener las personas luego de recibir un trato injusto.

Respecto a lo anterior, se han evidenciado diversas formas en que las personas enfrentan las situaciones de trato injusto. Cuando es habitual, se le denomina estilo de afrontamiento. Este concepto se refiere al tipo de estrategias adoptadas por las personas para hacerle frente a una situación estresante a partir de la evaluación cognitiva acerca de esta: modificar la situación, aceptar, buscar información, demorar la respuesta, etc. (26). En este sentido, se han desarrollado diversas escalas que miden este constructo, por ejemplo, el Inventario Multidimensional de Estimación de Afrontamiento, la Escala de Estilos y Estrategias de Afrontamiento, la Escala de Estrategias de Coping y el Inventario de Estrategias de Afrontamiento (27-30).

Un par de las ventajas de los instrumentos mencionados es que son de fácil administración y puntuación y que se utilizan en diversos contextos; sin embargo, existe escasa referencia de instrumentos que presenten un mayor grado especificidad y que evalúen dentro del 
constructo estilos de afrontamiento las reacciones de las personas según el trato que reciben en los sistemas de salud.

Considerando la importancia de las reacciones frente a una mala experiencia en la atención sanitaria y su impacto en las conductas de salud, así como la escasez de instrumentos que midan este constructo, un estudio en Chile se planteó una escala que permite valorar las reacciones que tienen las personas frente a situaciones de trato injusto o discriminatorio, la cual se desarrolló por medio de un proceso bottom up-top down y es culturalmente pertinente para la realidad chilena (31).

El proceso bottom up se refiere a indagar y describir desde las mismas personas, por medio de entrevistas u otras técnicas, los significados asociados a los constructos que se están estudiando. Entre tanto, la fase top down hace referencia a la aplicación de instrumentos. En la fase bottom up se realizaron entrevistas a 32 personas con diversas características sociodemográficas en cuanto a sexo (hombre y mujer), sistema de salud en que se atiende (público o privado), etnicidad (mapuche o no mapuche) y edad. En las entrevistas se les preguntaba a las personas respecto de las consecuencias y reacciones que han tenido producto de un trato injusto. Las respuestas se transcribieron y, posteriormente, la información se trianguló para cumplir con el criterio de rigor de comprobabilidad. Luego, se sometió a análisis de contenido simple, del cual se extrajeron los principales contenidos, los que se organizaron en categorías. Con las de mayor frecuencia se desarrollaron ítems de respuesta cerrada y tipo Likert. La escala resultante contenía 15 reactivos, que pasaron por una evaluación de su coherencia y suficiencia a través del juicio de 10 jueces expertos. Así, quedó conformada por 13 ítems. En función de lo anterior, se realizaron modificaciones que resultaron en el instrumento presentado en este estudio. En consecuencia, este artículo da cuenta de la fase top down del instrumento.

El presente estudio, entonces, se propuso como objetivos: 1) estimar las propiedades psicométricas de este instrumento, llamado Escala de Reacciones ante Experiencias de Trato Desigual en Salud (Eretrades), de Baeza-Rivera, y 2) establecer si existen diferencias estadísticamente significativas en las reacciones que experimentan las personas a partir del sistema en que se atienden y la pertenencia o no a la etnia mapuche (31).

\section{Materiales y métodos}

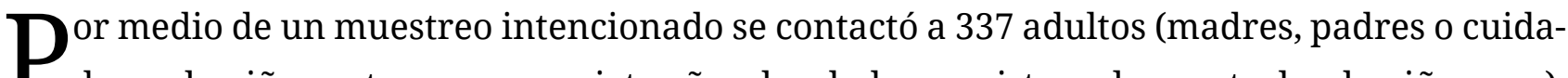
dores de niños entre un mes y siete años de edad que asisten a los controles de niño sano). Como criterio de inclusión se consideró que hubiesen tenido, al menos, una experiencia en los sistemas de salud con médico para la atención de su hijo/a. 
El promedio de edad de los participantes fue de 31 años $(D E=7.46)$ y de los hijos/as fue de 25 meses $(D E=17.01)$. La mayoría de quienes contestaron fueron mujeres $(85 \%)$. Un $57 \%$ reportó ser usuario del sistema público de salud y un $32 \%$ declaró pertenecer a la etnia mapuche. Las características de la muestra se observan en la tabla 1, con datos demográficos de los participantes, entre ellos edad, sexo, procedencia, sistema de salud en el que se atiende, nivel educacional, ingreso, nivel socioeconómico de acuerdo con la escala Esomar (32) y etnia.

Tabla 1. Características de la muestra

\begin{tabular}{|c|c|}
\hline Variable & Porcentaje \\
\hline \multicolumn{2}{|l|}{ Sexo } \\
\hline Mujer & 85 \\
\hline Hombre & 15 \\
\hline \multicolumn{2}{|l|}{ Sistema de salud } \\
\hline Público & 57 \\
\hline Privado & 43 \\
\hline \multicolumn{2}{|l|}{ Etnia } \\
\hline No mapuche & 68 \\
\hline Mapuche & 32 \\
\hline \multicolumn{2}{|l|}{ Estado civil } \\
\hline Casada/o & 44 \\
\hline Soltera/o & 30 \\
\hline Conviviendo & 22 \\
\hline \multicolumn{2}{|l|}{ Nivel educacional } \\
\hline Básica completa & 10 \\
\hline Media completa o técnica incompleta & 33 \\
\hline Universitaria & 48 \\
\hline Posgrado & 9 \\
\hline \multicolumn{2}{|c|}{ Ingreso mensual (pesos chilenos) } \\
\hline $0-500000$ & 64 \\
\hline $501000-1000000$ & 21 \\
\hline $1000001-1500000$ & 10 \\
\hline Sobre 1500000 & 5 \\
\hline
\end{tabular}

La Eretrades, de Baeza-Rivera, contiene 13 reactivos que evalúan el grado de acuerdo con las posibles reacciones experimentadas a partir de la percepción de haber recibido un trato desigual en contextos de salud (31). Un ejemplo de ítem es "Se desmotivó a llevar a su niño/a a los controles”. Para cada ítem existen cinco opciones de respuesta, las que se puntúan en una 
escala tipo Likert que va desde 1 = muy en desacuerdo a 5 = muy de acuerdo. Las propiedades psicométricas de la escala se pueden observar en la sección de "Resultados".

Los participantes se contactaron en los centros de salud familiar y jardines infantiles de la comuna de Temuco (Chile), previa autorización del Departamento de Salud Municipal de Temuco y de la Junta Nacional de Jardines Infantiles (entidad que agrupa a los jardines o guarderías infantiles). Los participantes firmaron el consentimiento informado aprobado por el Comité de Ética del Servicio de Salud Araucanía Sur y, posteriormente, contestaron el instrumento. Por último, firmaron un recibo simple por la retribución económica recibida por el tiempo dedicado a la investigación.

Los datos se analizaron con el programa estadístico spss versión 20.0. En primer lugar, la muestra se caracterizó con análisis descriptivos. Posteriormente, se llevó a cabo el análisis factorial exploratorio, para evaluar la estructura factorial del instrumento. Para ello, se evaluó la capacidad de factorizar de la matriz, por medio de las medidas de adecuación muestral de Kaiser-Meyer-Olkin (кмо) y la prueba de Bartlett, esperando resultados mayores a 0.80 para кмо, у así rechazar la hipótesis nula respecto de que la matriz es de identidad para Bartlett $(33,34)$. Después se llevó a cabo un análisis factorial exploratorio, utilizando el método máxima verosimilitud y rotación oblimin, con el cual se buscó la mejor solución, considerando criterios estadísticos y teóricos, como: a) seleccionar solo los factores que tuvieran autovalores mayores a uno, b) eliminar los ítems cuya carga factorial fuera menor a 0.30 y c) eliminar los factores compuestos por menos de tres ítems. Una vez identificados los factores, se les designó un nombre. Luego, para evaluar la confiabilidad de las escalas, se realizaron los análisis de consistencia interna por medio del a de Cronbach (35). Finalmente, con el análisis de varianza (Anova) se estableció si existen diferencias de medias en los diferentes tipos de reacciones, de acuerdo con el sistema de salud en que se atiende a la persona y la pertenencia o no a la etnia mapuche.

\section{Resultados}

I os resultados dan cuenta de una matriz factorizable. Específicamente, la prueba кмо fue estructura factorial da cuenta de dos factores: el primero se denominó reacciones evitativas del cuidado de la salud, y el segundo factor, reacciones de confrontación en el cuidado de la salud. Ambos factores explican el $59.7 \%$ de la varianza. No se eliminaron ítems. La composición de la escala, las cargas factoriales de los ítems, junto con la varianza explicada, el índice de confiabilidad, los promedios y desviaciones estándar de los ítems que componen la escala, se pueden apreciar en la tabla 2. 
Tabla 2. Estructura factorial, confiabilidad, promedio y desviaciones estándar de los ítems de la Escala de Reacciones ante Experiencias de Trato Desigual en Salud

\begin{tabular}{|c|c|c|c|c|}
\hline n. ${ }^{\circ}$ & Ítem & $\begin{array}{l}\text { Reacciones } \\
\text { evitativas }\end{array}$ & $\begin{array}{l}\text { Reacciones de } \\
\text { confrontación }\end{array}$ & $\begin{array}{l}\text { Promedio } \\
\text { (DT) }\end{array}$ \\
\hline 3 & Si pudiera cambiaría de centro de salud & 0.85 & & $2.78(1.56)$ \\
\hline 1 & $\begin{array}{l}\text { Preferiría que su hijo/a no se atendiera más con ese } \\
\text { profesional }\end{array}$ & 0.82 & & $3.10(1.61)$ \\
\hline 4 & Sintió rechazo hacia los médicos/enfermeras & 0.83 & & $2.53(1.38)$ \\
\hline 5 & Les contó lo sucedido a su familia y otras personas & 0.81 & & $3.28(1.59)$ \\
\hline 2 & Se desmotivó a llevar a su hijo/a a los controles & 0.81 & & $2.39(1.40)$ \\
\hline 7 & Postergó o demoró más en ir a la próxima consulta & 0.69 & & $2.15(1.23)$ \\
\hline 6 & No siguió las instrucciones que le dieron & 0.67 & & $2.04(1.16)$ \\
\hline 11 & Puso un reclamo con el jefe del profesional & & 0.80 & $1.92(1.27)$ \\
\hline 9 & $\begin{array}{l}\text { Reclamó a través de medios de comunicación (radio, } \\
\text { diario, Facebook, etc.) }\end{array}$ & & 0.73 & $1.69(1.10)$ \\
\hline 12 & Reclamó directamente con el profesional & & 0.58 & $2.30(1.47)$ \\
\hline 8 & Insultó a los funcionarios que lo atendieron & & 0.57 & $1.64(1.00)$ \\
\hline 13 & Se negó a ser atendido por ese profesional & & 0.56 & $2.27(1.46)$ \\
\hline 10 & Prefirió medicar por su cuenta a su hijo/a & & 0.35 & $1.73(1.08)$ \\
\hline & Promedio (DT) & $2.61(1.12)$ & $1.92(0.91)$ & \\
\hline & Varianza explicada & $41.4 \%$ & $18.3 \%$ & \\
\hline & a de Cronbach & 0.902 & 0.823 & \\
\hline
\end{tabular}

Como se mencionó, el primer factor se denominó reacciones evitativas, ya que alude a respuestas más bien pasivas o de evitación frente a una situación de maltrato, que no involucran un enfrentamiento directo con quien ejerció la ofensa; por ejemplo, cambiar de centro de salud. Al segundo factor se le llamó reacciones de confrontación, ya que, al contrario del primero, estas aluden a reclamos directos o manifestaciones externas o hacia terceros por la situación vivida.

La media en ambos factores es baja; sin embargo, en promedio, las personas tienden hacia más conductas evitativas que de confrontación. Se identificaron diferencias estadísticamente significativas entre ambos tipos de reacciones, con un tamaño del efecto medio $(t=11.54 ; p<0.001 ; d$ de Cohen $=0.67)$.

$\mathrm{El}$ análisis de correlación entre los factores indica que se encuentran altamente asociados $(r=0.642 ; p<0.001)$, la correlación es directa y su magnitud es alta, es decir a mayores reacciones evitativas, más reacciones de confrontación.

Finalmente, se procedió a realizar un Anova factorial con las variables etnia y sistema en que se atiende. Los resultados dan cuenta de que, en ambos factores, la etnia, por sí sola, no tiene efecto; pero sí el sistema de salud en que se atiende y la interacción entre ambas variables, tal 
como se aprecia en las tablas 3 y 4 . Es decir, las personas mapuches que se atienden en el sistema público presentan mayor promedio que las no mapuches, tanto en las reacciones evitativas como en las de confrontación. Mientras que en el sistema privado, las personas no mapuches tienen mayor promedio en ambos factores, en comparación con los mapuches.

Tabla 3. Descriptivos (promedios y desviaciones típicas) para etnia y sistema

\begin{tabular}{lllll}
\hline & & $\begin{array}{c}\text { Reacciones } \\
\text { evitativas }\end{array}$ & $\begin{array}{c}\text { Reacciones de } \\
\text { confrontación }\end{array}$ & $\begin{array}{c}\text { Promedio } \\
\text { escala total }\end{array}$ \\
\hline \multirow{2}{*}{ Sistema público } & Mapuche & $2.97(0.93)$ & $2.15(0.89)$ & $2.58(0.80)$ \\
& No mapuche & $2.44(1.15)$ & $1.81(0.87)$ & $2.15(0.92)$ \\
Sistema privado & Mapuche & $1.70(1.13)$ & $1.23(0.65)$ & $1.48(0.82)$ \\
& No mapuche & $2.67(1.17)$ & $1.97(0.98)$ & $2.34(0.98)$ \\
\hline
\end{tabular}

Tabla 4. Anova factorial para las variables sistema en que se atiende y etnia

\begin{tabular}{lllrrr}
\hline & & $\boldsymbol{g}$ & $\boldsymbol{F}$ & $\boldsymbol{p}$ & $\boldsymbol{\eta}_{\mathbf{p}}{ }^{2}$ \\
\hline \multirow{3}{*}{ Reacciones evitativas } & Sistema (S) & 1 & 5.32 & $<0.05$ & 0.03 \\
& Etnia (E) & 1 & 0.97 & 0.33 & 0.01 \\
& $\mathrm{~S} \times \mathrm{E}$ & 1 & 10.97 & $<0.01$ & 0.05 \\
\hline \multirow{5}{*}{ Reacciones de confrontación } & Sistema (S) & 1 & 4.22 & $<0.05$ & 0.02 \\
& Etnia (E) & 1 & 1.18 & 0.28 & 0.01 \\
& $\mathrm{~S} \times \mathrm{E}$ & 1 & 8.42 & $<0.01$ & 0.04 \\
\hline \multirow{3}{*}{ Escala total } & Sistema (S) & 1 & 5.91 & 0.16 & 0.027 \\
& Etnia (E) & 1 & 1.29 & 2.56 & 0.006 \\
& $\mathrm{~S} \times \mathrm{E}$ & 1 & 11.99 & $<0.01$ & 0.054 \\
\hline
\end{tabular}

\section{Discusión}

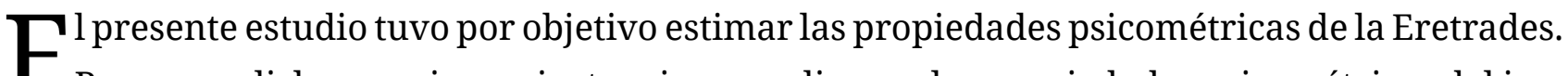
EPara cumplirlo, en primera instancia se analizaron las propiedades psicométricas del instrumento y, luego, se compararon los resultados en función de la etnia y el sistema en que se atiende la persona. En función de lo planteado, en este estudio se cumplen los objetivos propuestos.

Como resultado de este proceso, la versión final del instrumento presentó una estructura bifactorial y adecuadas propiedades psicométricas para ser utilizado en el ámbito de la salud. El primer factor, reacciones evitativas, alude mecanismos de afrontamiento pasivos, en que 
el paciente opta o bien por prescindir de las atenciones médicas, o bien comparte la experiencia de trato injusto con su círculo cercano; mientras que el segundo factor, reacciones de confrontación, corresponde a la manifestación directa de descontento hacia el profesional de la salud, así como a instancias de jerarquías más altas.

Es importante mencionar que ambos factores se encuentran directamente relacionados; por tanto, en la práctica aquellos quienes tienen reacciones evitativas frente al trato injusto tienen altas probabilidades de evidenciar conjuntamente reacciones de confrontación. Además, se observó una tendencia a manifestar en mayor medida reacciones evitativas que de confrontación.

Pese a que se requiere evidencia adicional para comprender lo expuesto, se hipotetiza que, probablemente, estas reacciones son dependientes de la percepción de trato injusto, donde situaciones más intensas, que podrían generar una mayor percepción de trato injusto, estarían asociadas a respuestas complejas, en que se evidencien tanto reacciones pasivas como de confrontación. Por el contrario, experiencias con una menor percepción de trato injusto se vincularían con reacciones pasivas sin que la persona llegue a desplegar reacciones de confrontación. Esto se relacionaría con las emociones que se experimentan como consecuencia del trato injusto, cuando emociones más displacenteras y que tienen mayor poder de activación tienden a movilizar a las personas a actuar (36).

Por otra parte, el hecho de que el factor de reacciones evitativas tenga un promedio mayor, da cuenta de que en general las personas tienden a expresar y compartir su malestar con sus cercanos, es decir, socializan la experiencia de manera que esto se transmite hacia terceros, y ello genera opiniones y contribuye a la formación de creencias socialmente compartidas respecto de las atenciones en salud. Estas creencias pueden predisponer a los usuarios a enfrentar futuros encuentros clínicos.

Además, se hipotetiza que las personas presentan cierto grado de temor a reaccionar de manera confrontacional, por miedo a que el personal sanitario tome represalias en contra de ellos o sus familiares en las próximas atenciones de salud, por lo que se opta por compartir las experiencias con los cercanos, más que confrontar directamente al profesional de la salud o a las autoridades correspondientes, sobre todo considerando que en el sistema público de salud la posibilidad de cambiar de prestador no es tan fácil como en el sistema privado, donde es posible escoger con quién atenderse (37).

Aun cuando en Chile existe la Ley de Derechos y Deberes de los Pacientes n. ${ }^{\circ} 20.584$ de 2012, la cual expresa que las personas deben recibir un trato digno, atención de calidad y segura, consultar o reclamar respecto de la atención de salud recibida, entre otros aspectos, no siempre ocurre en la realidad ni es es exigido por la población.

Ahora bien, al analizar lo anterior a la luz de las variables "sistema de salud en que se atiende el usuario" y "etnia”, llama la atención que el subgrupo con los puntajes más elevados fue el de usuarios de la etnia mapuche que se atienden en el sistema público; por 
el contrario, el que mostró las puntuaciones más bajas fue el mapuche que se atiende en el sistema privado. Lo anterior puede deberse al escaso número de participantes mapuche que se atienden en el sistema privado de salud. Específicamente, en el sistema público se atiende el $86.4 \%$ de la población que se identifica como indígena y el $77.2 \%$ de quienes no se identifican como indígenas (38).

Además, el sistema público, en comparación con el sistema privado, posee una peor evaluación en términos de su calidad y atención (8). Al respecto, un estudio desarrollado por Baeza-Rivera et al. da cuenta de que existe una desvalorización hacia el sistema público por quienes se atienden en él y, por el contrario, una sobrevaloración del sistema privado, lo que se asociaría a la creencia de que el pagar por la atención de salud supone un mejor servicio, lo que no necesariamente ocurre en la realidad (37).

Respecto a las personas del pueblo mapuche, es importante mencionar que están expuestas a mayor discriminación, tanto por la pertenencia a su etnia como por pertenecer, en general, a niveles socioeconómicos y educacionales más bajos $(39,40)$. Esta discriminación acumulativa expone a los mapuches a experiencias cotidianas de discriminación en diversos aspectos de su vida (incluido el ámbito de la salud), lo que influye de modo negativo en distintos indicadores de salud y genera un círculo vicioso de desigualdad (16). De esta manera, pudiera ser que las personas mapuches tienden a estar más sensibles a percibir malos tratos, ya que históricamente han sido discriminadas y han sido víctimas de situaciones de trato injusto, especialmente en las atenciones de salud (41).

Esta investigación presenta ciertas ventajas, como utilizar un diseño en etapas sucesivas, que permitió, en primera instancia, levantar datos de los contenidos emanados de la población local, para después construir una escala culturalmente pertinente. Por último, se pusieron a prueba sus propiedades psicométricas con una muestra de pacientes de ambos sistemas de salud.

En línea con lo anterior, además, se cuenta con buen tamaño muestral, pese a las dificultades que supone acceder a pacientes de los servicios de salud. Sin embargo, presenta algunas limitaciones, como el bajo porcentaje de hombres que participó, lo cual se puede explicar porque el cuidado de los niños, en general, ha sido delegado a las mujeres, por lo que integrar las reacciones experimentadas por los hombres frente a los malos tratos en salud y estimar si existen diferencias por género puede enriquecer futuras investigaciones. Una segunda limitación es que hubo un porcentaje menor de personas mapuche que se atendía en el sistema de salud privado, lo que está dado principalmente por la distribución de la población en los sistemas de salud.

Con todo lo anterior, se obtuvo una escala con buenas propiedades psicométricas, de fácil uso y puntuación. Esta escala representa un aporte en la comprensión de un fenómeno que hasta ahora ha sido estudiado casi exclusivamente desde un enfoque biomédico; además, casi sin atención a la adaptación cultural de los instrumentos utilizados. Por tanto, esta escala 
responde a la necesidad de contar con instrumentos que midan las reacciones derivadas del trato injusto en el contexto local.

Este instrumento puede utilizarse en diversos contextos de salud, porque es útil para comprender desde una mirada psicológica el proceso de salud-enfermedad, identificando las conductas asociadas a percibir un mal trato, lo que se relacionaría con consecuencias de salud física y mental $(15,16)$.

A modo de conclusión, este tipo de estudios entrega evidencia para el uso de instrumentos en el ámbito de la salud. Contar con ellos permite extenderlos para evaluar las consecuencias que los malos tratos tienen en las conductas y cómo esto puede repercutir en la salud de las personas, considerando que tanto la motivación como la forma de afrontar las situaciones son predictores del bienestar subjetivo y psicológico de las personas y se ven influenciados por la capacidad de controlar la situación y la susceptibilidad a cambiarlas $(42,43)$.

\section{Agradecimientos}

Ele estudio fue parte de la tesis doctoral de la Dra. María José Baeza-Rivera, desarrollada en de Investigación Científica y Tecnológica, número de folio 21140104.

\section{Contribución de los autores}

María José Baeza-Rivera: revisión bibliográfica, colección de datos, análisis de los datos, preparación del manuscrito, revisión y corrección del manuscrito.

Natalia Salinas-Oñate: diseño del estudio, revisión bibliográfica, recolección de datos, preparación y corrección del manuscrito.

Neli Escandón-Nagel: análisis de los datos, preparación del manuscrito, revisión y corrección del manuscrito.

Esteban Caamaño Mardones: preparación del manuscrito, revisión y corrección del manuscrito.

\section{Descargos de responsabilidad}

F sta investigación fue financiada por la Comisión Nacional de Investigación Científica y ETecnológica, Beca Doctorado Nacional, número de folio 21140104. 


\section{Conflicto de intereses}

Ninguno declarado.

\section{Referencias}

1. Banco Mundial. Indicadores de desarrollo mundial: Chile [internet]. Disponible en: https://datos.bancomundial.org/pais/chile?view=chart

2. Méndez CA, Vanegas López JJ. La participación social en salud: el desafío de Chile. Rev Panam Salud Pública. 2010;27(2):144-8.

3. Subsecretaría de Redes Asistenciales, División de Gestión de la Red Asistencial de Chile. Objetivos sanitarios para la década 2000-2010: evaluación a mitad de cumplimiento. Evaluación Objetivo III. Disminuir las desigualdades en salud [internet]. Santiago de Chile; 2006. Disponible en: http://www.bibliotecaminsal.cl/wp/wp-content/uploads/2016/03/5.pdf

4. Ministerio de Salud de Chile. Norma técnica para la supervisión de niños y niñas de 0 a 9 años en la atención primaria de salud [internet]. Valparaíso; 2013. Disponible en: http://www.centroucdown.uc.cl/images/PDF/norma-tecnica-supervision-ninos-ninas_ compressed.pdf

5. Szot J. Reseña de la salud pública materno-infantil chilena durante los últimos 40 años: 1960-2000. Rev Chil Obstet Ginecol. 2002;67(2):129-35.

6. Alarcón AM, Vidal AC. Dimensiones culturales en el proceso de atención primaria infantil: perspectivas de las madres. Salud Pública Mex. 2005;47(6):440-6.

7. Pezoa M. Satisfacción y calidad percibida en la atención de salud hospitalaria [internet]. Santiago de Chile; 2013. Disponible en: http://www.supersalud.gob.cl/documentacion/666/articles-9005_recurso_1.pdf

8. Ministerio de Salud de Chile, Departamento de Estadísticas e Información en Salud. Información [internet]. 2014. Disponible en: https://deis.minsal.cl/

9. Ortiz PM, Ortiz PE. Psicología de la salud: una clave para comprender el fenómeno de la adherencia terapéutica. Rev Méd Chil. 2007;135(5). https://doi.org/10.4067/S003498872007000500014

10. Brondolo E, Love EE, Pencille M, Schoenthaler A, Ogedegbe G. Racism and hypertension: a review of the empirical evidence and implications for clinical practice. Am J Hypertens. 2011 1;24(5):518-29. https://doi.org/10.1038/ajh.2011.9

11. Levine DS, Himle JA, Abelson JM, Matusko N, Dhawan N, Taylor RJ. Discrimination and social anxiety disorder among African-Americans, Caribbean Blacks, and non-hispanic whites. J Nerv Ment Dis. 2014;202(3):224-30.

12. McLaughlin KA, Hatzenbuehler ML, Keyes KM. Responses to discrimination and psychiatric disorders among black, hispanic, female, and lesbian, gay, and bisexual individuals. Am J Public Health. 2010 Aug;100(8):1477-84. https://doi.org/10.2105/AJPH.2009.181586 
13. Soto JA, Dawson-Andoh NA, BeLue R. The relationship between perceived discrimination and generalized anxiety disorder among African Americans, Afro Caribbeans, and non-hispanic whites. J Anxiety Disord. 2011;25(2):258-65. https://doi.org/10.1016/j. janxdis.2010.09.011

14. Taylor TR, Kamarck TW, Shiffman S. Validation of the Detroit area study discrimination scale in a community sample of older African American adults: the Pittsburgh Healthy Heart Project. Int J Behav Med. 2004;11(2):88-94. https://doi.org/10.1207/ s15327558ijbm1102_4

15. Marinho ML. Health inequity in a neoliberal society: lifestyle choices or constrained practices? Warwick University; 2015.

16. Ortiz MS, Baeza-Rivera MJ, Salinas-Oñate N, Flynn P, Betancourt H. Atribución de malos tratos en servicios de salud a discriminación y sus consecuencias en pacientes diabéticos mapuche. Rev Méd Chil. 2016;144(10):1270-6. https://doi.org/10.4067/S003498872016001000006

17. Bird ST, Bogart LM, Delahanty DL. Health-related correlates of perceived discrimination in HIV care. AIDs Patient Care sTDs. 2004;18(1):19-26. https://doi. org/10.1089/108729104322740884

18. Burgess D, Ding Y, Hargreaves M, van Ryn M, Phelan S. The association between perceived discrimination and underutilization of needed medical and mental health care in a multi-ethnic community sample. J Health Care Poor Underserved. 2008;19(3):894911. https://doi.org/10.1353/hpu.0.0063

19. Hausmann LRM, Jeong K, Bost JE, Ibrahim SA. Perceived discrimination in health care and health status in a racially diverse sample. Med Care. 2008;46(9):905-14.

20. Smedley BD, Myers HF. Conceptual and methodological challenges for health disparities research and their policy implications. J Soc Issues. 2014;70(2):382-91. https://doi. org/10.1111/josi.12065

21. Trivedi AN, Ayanian JZ. Perceived discrimination and use of preventive health services. J Gen Intern Med. 2006;21(6):553-8. https://doi.org/10.1111/j.1525-1497.2006.00413.x

22. Bazargan-Hejazi S, Bing E, Bazargan M, Der-Martirosian C, Hardin E, Bernstein J, et al. Evaluation of a brief intervention in an inner-city emergency department. Ann Emerg Med. 2005;46(1):67-76.

23. Baeza-Rivera MJ, Betancourt H, Salinas-Oñate N, Ortiz MS. Creencias culturales sobre los médicos y percepción de discriminación: el impacto en la continuidad de la atención. Rev Méd Chil. 2019;147(2):161-7. https://doi.org/10.4067/s0034-98872019000200161

24. Ministerio de Desarrollo Social. Informe de desarrollo social. Santiago de Chile; 2018.

25. Ministerio de Planificación del Gobierno de Chile. Encuesta de Caracterización Socioeconómica Nacional 2009 (CASEN) [internet]. Santiago de Chile; 2009. Disponible en: http://pnre.ine.cl/index.php/catalog/85

26. Solís Manrique C, Vidal Miranda A. Estilos y estrategias de afrontamiento en adolescentes. Rev Psiquiatr Salud Ment Hermilio Vald. 2006;7(1):33-9. 
27. Carver CS, Scheier MF, Weintraub JK. Assessing coping strategies: A theoretically based approach. J Pers Soc Psychol. 1989;56(2):267-83. https://doi.org/10.1037/00223514.56.2.267

28. Martín Díaz MD, Jiménez Sánchez MP, Fernández-Abascal E. Estudio sobre la escala de estilos y estrategias de afrontamiento ( $\left.E^{3} A\right)$. Rev Electrón Motiv Emoc. 2000;3(4).

29. Chorot P, Sandín B. Escala de Estrategias de Coping Revisado (EEC-R). Madrid: Uned; 1993.

30. Tobin DL, Holroyd KA, Reynolds R V., Wigal JK. The hierarchical factor structure of the coping strategies inventory. Cognit Ther Res. 1989;13(4):343-61.

31. Baeza-Rivera M. Discriminación percibida y sus consecuencias en las conductas de cuidado de la salud: un estudio multifactorial. Temuco: Universidad de La Frontera; 2015.

32. Adimark. El nivel socio económico Esomar: manual de aplicación [internet]. Santiago de Chile; 2000. Disponible en: https://www.microweb.cl/idm/documentos/ESOMAR.pdf

33. Kaiser HF. A second generation Little jiffy. Psychometrika. 1970;35(4):401-15.

34. Bartlett MS. Tests of significance in factor analysis. Br J Stat Psychol. 1950;3(2):77-85.

35. Cronbach LJ. Coefficient alpha and the internal structure of tests. Psychometrika. 1951;16:297-334.

36. Russell JA. A circumplex model of affect. J Pers Soc Psychol. 1980;39(6):1161-78.

37. Baeza-Rivera M, Valle G, Espinosa D, Guarderas M, Moreno A, Salinas-Oñate N, et al. Creencias culturales sobre el sistema y proveedores de la salud: una aproximación cualitativa. Rev Psicol Soc y Pers. 2016;XXXII(2):1-17.

38. Ministerio Desarrollo Social. Salud: Síntesis de Resultados. 2018.

39. Otzen T, Betancourt H, González-Plitt M-E, Martella D. Fatalism, attributions of failure and academic performance in mapuche and non-Mapuche Chilean students. An Psicol. 2016;32(2):341-8.

40. Quilaqueo D, Merino MR, Saiz JL. Representación social mapuche e imaginario social no mapuche de la discriminación percibida. Atenea (Concepc). 2007;496:81-103. https:// doi.org/10.4067/S0718-04622007000200006

41. Alarcón AM, Vidal A, Neira Rozas J. Salud intercultural: elementos para la construcción de sus bases conceptuales. Rev Méd Chil. 2003;131(9):1061-5.

42. Sanjuán P, Ávila M. Afrontamiento y motivación como predictores del bienestar subjetivo y psicológico. Rev Psicopatol Psicol Clín. 2016;21(1):1-10. https://doi.org/10.5944/ rppc.vol.21.num.1.2016.15401

43. Quezada-Berumen L, Moral de la Rubia J, Ibarra-González LD, González-Ramírez MT. Estudio de validación del Cuestionario de Afrontamiento del Estrés en personas trans de México. Rev Psicopatología y Psicol Clínica. 2018;23(2):121-34. https://doi.org/10.5944/ rppc.vol.23.num.2.2018.19367 